\title{
Periodic Green's Function for Skewed 3-D Lattices Using the Ewald Transformation
}

\author{
Ivica Stevanović and Juan R. Mosig \\ Laboratory of Electromagnetics and Acoustics (LEMA) \\ Ecole Polytechnique Fédérale de Lausanne (EPFL) \\ ELB, Station 11 \\ CH-1015 Lausanne \\ Switzerland \\ E-mails : Ivica.Stevanovic@epfl.ch, Juan.Mosig@epfl.ch \\ Phone : +41216934637 \\ Fax : $\quad$ +41216932673
}

Keywords: Ewald transformation, Green's functions (GFs), integral equations (IEs), periodic structures, electromagnetic/photonic band-gap (EBG/PBG) materials, metamaterials (MTMs).

\begin{abstract}
In this paper, we apply the Ewald acceleration technique to the efficient evaluation of periodic Green's functions (GFs) for 3-D skewed lattices like those arising in electromagnetic/photonic band-gap (EBG/PBG) structures and metamaterials. We develop the expression for the optimal value of an associated splitting parameter, derive the gradient of the scalar potential GFs and address the extraction of singularity for both vector/scalar potential GF and their curl/gradient. Several numerical implementation issues are also discussed leading to further enhancement in computational speed, accuracy, and numerical stability. Finally, the accuracy of the developed GFs is verified against well established algorithms.
\end{abstract}




\section{INTRODUCTION}

Periodic structures have a long history of being utilized to control electromagnetic scattering and radiation for various scientific and engineering applications. Examples include electromagnetic or photonic band-gap (EBG/PBG) structures [1] and left-handed metamaterials (MTMs) for which both the effective permittivity and permeability are negative [2]. In integral-equation (IE) analysis of periodic structures, efficient computation of the Green's functions (GFs) is of paramount interest. Since these structures are inspired by the three-dimensional (3-D) geometry of both natural crystals and those artificial crystals that can arise only in the human imagination [1], not only simple cubic lattices (with orthogonal primitive vectors), but also the ones with skewed lattices (such as facecentered cubic or diamond structures) need to be efficiently taken into account. In this paper we address the evaluation of GFs for periodic structures with such general lattices.

Among different techniques used to speed up the computation of Green's functions [3], the Ewald transform [4] has clearly demonstrated its suitability for periodic problems. It has been advantageously used in the efficient evaluation of GFs of infinite periodic phased arrays of line sources (2-D structures with 1-D periodicity) [5], [6], 2-D structures with 2-D periodicity [7], in 3D problems with 2-D orthogonal [8], [9] and skewed [10] lattices, as well as for rectangular cavities (3-D problems with 3-D orthogonal lattices) [11], [12]. Its application to 3-D non-orthogonal lattices has been first reported in [13], where the method is used to compute the energy bands for Schrödinger's equation.

In this paper, we present the Ewald sums for 3-D non-orthogonal (skewed) lattices used to solve 3D Helmholtz equation needed for the computation of electromagnetic bands of triply periodic structures such as electromagnetic (photonic) band-gaps and metamaterials. We develop the expression for the optimal value of the splitting parameter for general 3-D skewed lattices and show that it reduces to the formula presented in [14] for the case of orthogonal, nearly cubic 3-D lattices. Moreover, we derive the gradient of the scalar potential GFs and address the extraction of singularity for both vector/scalar potential GF and their curl/gradient. This is a mandatory step when solving periodic printed structures (infinite arrays) with a mixed potential integral equation (MPIE). The method can be used in conjunction with the integral-equations solved by method of moments (IE-MoM) to determine dispersion diagrams of 3-D metallo-dielectric structures.

\section{3-D SKEWED LATTICE SUMS}

The Green's function for point sources arranged in a 3-D lattice with a translation vector $\mathbf{r}_{\text {mn }}=m \mathbf{a}_{1}+n \mathbf{a}_{2}+p \mathbf{a}_{3}$ obeys the inhomogeneous Helmholtz equation

$$
\left(\nabla^{2}+k^{2}\right) \Psi(\mathbf{R})=\sum_{m, n, p} \delta\left(\mathbf{R}-\mathbf{r}_{m n p}\right) \mathrm{e}^{-\mathrm{j} \mathbf{k} \cdot \mathbf{r}_{m n p}}
$$

and is given by

$$
\Psi(\mathbf{R})=\frac{1}{4 \pi} \sum_{m, n, p} \frac{\mathrm{e}^{-\mathrm{j} k R_{m n p}}}{R_{m n p}} \mathrm{e}^{-\mathrm{j} \mathbf{k} \cdot \mathbf{r}_{m p}}
$$

where $\mathbf{R}=\mathbf{r}-\mathbf{r}^{\prime}$ is the source-observer distance within a primitive cell, $\mathbf{k}$ is the wave vector of the medium, $R_{m n p}=\left|\mathbf{R}-\mathbf{r}_{m n p}\right|$, and $\mathbf{a}_{i}, i=1,2,3$ are the primitive vectors of the direct lattice. The 
representation (2) is usually called the image formulation of the periodic GF. Applying to it the Poisson summation formula, one obtains the modal representation of the same GF

$$
\Psi(\mathbf{R})=\frac{1}{V} \sum_{m, n, p \neq 0} \frac{\mathrm{e}^{\mathbf{j} \mathbf{K}_{m n p} \cdot \mathbf{R}}}{\gamma_{m n p}^{2}} .
$$

In the last expression, $V=\mathbf{a}_{1} \cdot\left(\mathbf{a}_{2} \times \mathbf{a}_{3}\right)$ is the volume of the primitive cell, $\mathbf{K}_{m n p}=\mathbf{k}_{m n p}-\mathbf{k}$, and $\gamma_{m n p}^{2}=\left|\mathbf{K}_{m n p}\right|^{2}-k^{2}$. The translation vector of the reciprocal lattice $\mathbf{k}_{m n p}=m \mathbf{k}_{1}+n \mathbf{k}_{2}+p \mathbf{k}_{3}$ has the primitive vectors defined by

with

$$
\mathbf{k}_{i} \cdot \mathbf{a}_{j}=2 \pi \delta_{i j}
$$

$$
\mathbf{k}_{1}=2 \pi \frac{\mathbf{a}_{2} \times \mathbf{a}_{3}}{\mathbf{a}_{1} \cdot\left(\mathbf{a}_{2} \times \mathbf{a}_{3}\right)}, \mathbf{k}_{2}=2 \pi \frac{\mathbf{a}_{3} \times \mathbf{a}_{1}}{\mathbf{a}_{1} \cdot\left(\mathbf{a}_{2} \times \mathbf{a}_{3}\right)}, \mathbf{k}_{3}=2 \pi \frac{\mathbf{a}_{1} \times \mathbf{a}_{2}}{\mathbf{a}_{1} \cdot\left(\mathbf{a}_{2} \times \mathbf{a}_{3}\right)} .
$$

Both infinite sums in (2) and (3) are slowly convergent. The image sum (2) converges faster when the observer point is in the source point region since the singularity is perfectly included in the expression. However, close to the lateral periodic walls, the convergence is deteriorated as the periodic boundary conditions (BCs) are going to be satisfied only for an infinite number of images taken into account. Each term of the modal sum (3), on the other hand, satisfies the BCs at the lateral walls and, hence, the convergence of this sum is faster close to the walls. However, taking into account the singularity at the source point would require an infinite number of modes leading, therefore, to the slow convergence of the modal sum in the source point region.

The Ewald method successfully combines both image and modal formulations into a fast converging series. Here, we present the details of the Ewald transform used to compute the potential GFs of a periodic structure with a general 3-D skewed lattice.

Following Ewald [3], we use the relation

$$
\frac{\mathrm{e}^{-\mathrm{j} k R}}{R}=\frac{2}{\sqrt{\pi}} \int_{0}^{\infty} \mathrm{e}^{-R^{2} \xi^{2}+\frac{k^{2}}{4 \xi^{2}}} \mathrm{~d} \xi
$$

where $\xi$ is a complex variable and the path of integration is chosen such that the integrand remains bounded as $\xi \rightarrow 0$ and decays $\xi \rightarrow \infty$ [8]. Breaking the integral into two parts $(0, E)$ and $(E, \infty)$, the GF $\Psi(\mathbf{R})$ can be expressed as

$$
\Psi(\mathbf{R})=\Psi_{1}(\mathbf{R})+\Psi_{2}(\mathbf{R})
$$

where

$$
\Psi_{1}(\mathbf{R})=\frac{1}{4 \pi} \sum_{m, n, p} \mathrm{e}^{-\mathbf{j k} \cdot \mathbf{r}_{m n p}} \frac{2}{\sqrt{\pi}} \int_{0}^{E} \mathrm{e}^{-R_{m n p} \xi^{2}+\frac{k^{2}}{4 \xi^{2}}} \mathrm{~d} \xi
$$

and

$$
\Psi_{2}(\mathbf{R})=\frac{1}{4 \pi} \sum_{m, n, p} \mathrm{e}^{-\mathrm{j} \mathbf{k} \cdot \mathbf{r}_{m p}} \frac{2}{\sqrt{\pi}} \int_{E}^{\infty} \mathrm{e}^{-R_{m m p} \xi^{2}+\frac{k^{2}}{4 \xi^{2}}} \mathrm{~d} \xi
$$

The Poisson summation formula and the shifting properties of Fourier transform may be used to transform (8) into 


$$
\Psi_{1}(\mathbf{R})=\frac{1}{V} \sum_{m, n, p \neq 0} \frac{\mathrm{e}^{-\frac{\gamma_{m p p}^{2}}{4 E^{2}}}}{\gamma_{m n p}^{2}} \mathrm{e}^{\mathrm{j} \mathbf{K}_{m m p} \cdot \mathbf{R}}
$$

A straightforward application of the following identity [15]

$$
\int_{r}^{\infty} \mathrm{e}^{-p^{2} \xi^{2}+\frac{q^{2}}{4 \xi^{2}}} \mathrm{~d} \xi=\frac{\sqrt{\pi}}{4 p} \sum_{ \pm} \mathrm{e}^{ \pm \mathrm{jpq}} \mathrm{erfc}\left(p r \pm \frac{\mathrm{j} q}{2 r}\right)
$$

to $\Psi_{2}(\mathbf{R})$, with $p=R_{m n p}>0, q=k$, and $r=E$ yields

$$
\Psi_{2}(\mathbf{R})=\frac{1}{8 \pi} \sum_{m, n, p} \mathrm{e}^{-\mathrm{j} \mathbf{k} \cdot \mathbf{r}_{m p}} \sum_{ \pm} \frac{\mathrm{e}^{ \pm \mathrm{j} k R_{m n p}}}{R_{m n p}} \operatorname{erfc}\left(R_{m n p} E \pm \frac{\mathrm{j} k}{2 E}\right)
$$

where $\sum_{ \pm}$designates the sum of the terms with + and - signs.

Both sums (10) and (12) have Gaussian convergence rate and accurate values are obtained for a very small number of terms if the parameter $E$ is properly chosen. The best choice for the splitting parameter is the one that balances the rate of decay of the two series, making both contribute an equal number of terms to the final value. The approximation to the optimal value of $E$ in $3-D$ skewed lattice will be derived in the following section.

The accurate numerical evaluation of the complex complementary error function can be done using very efficient numerical algorithms [16], [17]. A computationally efficient way of implementing GFs in a computer code is to pre-compute them at each frequency point and tabulate the values in a rectangular 3-D grid along $x-x^{\prime}, y-y^{\prime}$, and $z-z^{\prime}$. The GF values needed to compute the numerical integrals in the MoM matrix are then retrieved from the table using interpolation routines [17]. With a dense enough grid and nonuniform (cubic) distribution of points in the source region, the interpolation leads to very accurate results and much faster computation than if the GFs are evaluated directly.

\section{OPTIMUM SPLITTING PARAMETER FOR 3-D SKEWED LATTICES}

We analyze here the convergence properties of $\Psi_{1}$ and $\Psi_{2}$ given by (10) and (12), respectively. For large $m, n, p$, we can approximate $\gamma_{m n p} \approx k_{m n p}$, which yields the following asymptotic behavior of terms in $\Psi_{1}$

$$
\sim \exp \left(-\frac{k_{m n p}^{2}}{4 E^{2}}\right) /\left(\frac{k_{m n p}^{2}}{4 E^{2}}\right)
$$

In the image sum $\Psi_{2}$, for large $m, n, p$, we can approximate $R_{m n p}^{2} \approx r_{m n p}^{2}$. Moreover, the asymptotic expansion for the complementary error function for large arguments $\operatorname{erfc}(z) \sim \exp \left(-z^{2}\right) /(\sqrt{\pi} z)$ leads to the following asymptotic behavior of the terms in $\Psi_{2}$ :

$$
\sim \frac{\mathrm{e}^{-r_{m p}^{2} E^{2}}}{r_{m n p}^{2} E^{2}} \text {. }
$$


Observing the asymptotic behavior of the terms in both sums, we must set [6]

$$
r_{m n p}^{2} E^{2}=\frac{k_{m n p}^{2}}{4 E^{2}} \equiv \sigma^{2}
$$

in order to obtain the same number of significant digits of accuracy in each sum. The convergence rate in both sums is predominantly governed by the minimum absolute value of the three lattice vectors in either lattices $a_{\min }=\min \left(a_{1}, a_{2}, a_{3}\right)$ and $k_{\min }=\min \left(k_{1}, k_{2}, k_{3}\right)$. The maximum number of terms in the direction of the minimum lattice vector to achieve the given accuracy $\sigma$ can be expressed as $M_{k}=2 \sigma E / k_{\min }$ and $M_{a}=\sigma /\left(a_{\min } E\right)$ for modal and image formulations, respectively. The value of $E=E_{0}$ for which we have balanced sums is obtained for $M_{k}=M_{a}$ and given by

$$
E_{0}=\sqrt{\frac{k_{\min }}{2 a_{\text {min }}}} .
$$

For a simple cubic lattice, where $\left(\mathbf{a}_{1}, \mathbf{a}_{2}, \mathbf{a}_{3}\right)$ are mutually orthogonal, it can be shown that (16) reduces to the expression reported in [14]. Although the approximation from [14] performs well for nearly cubic lattices, in the case of general skewed lattices, (16) should be used instead.

As already noted in [6] and [14], for large periodic spacing, the imaginary part of the argument $\left(R_{m n p} E \pm \mathrm{j} k /(2 E)\right)$ in $\Psi_{2}$ can become very large and cause numerical instability since the complementary error functions in (12) asymptotically behave like

$$
\mathrm{e}^{-r_{m p p}^{2} E^{2}+\frac{k^{2}}{4 E^{2}}} \text {. }
$$

The same comments can be made for the series $\Psi_{1}$ that includes the Gaussian function directly and behaves like

$$
\mathrm{e}^{-\frac{k_{m m p}^{2}}{4 E^{2}}+\frac{k^{2}}{4 E^{2}}}
$$

Therefore, when the spacing, or equivalently the frequency is large, the two series $\Psi_{1}$ and $\Psi_{2}$ converge to very large values that are nearly equal in magnitude but of opposite signs and adding them leads to severe loss of accuracy due to a finite machine precision. The problem may be remedied by requiring $k^{2} /\left(4 E^{2}\right)<H^{2}$, where $H^{2}$ is the maximum exponent permitted. This yields the following choice of the optimum parameter

$$
E=\max \left(E_{0}, \frac{k}{2 H}\right) \text {. }
$$

In the case $E>E_{0}$, the number of terms needed for the modal sum to converge will be larger than the number of terms for which the convergence of the image sum is achieved.

\section{GRADIENT OF THE PERIODIC GF}

The electric and magnetic vector potentials in 3-D periodic structures are both diagonal dyadics. To calculate the electric field due to a magnetic current or the magnetic field due to an electric current, the curl of the vector potential GFs is needed. Additionally, $\nabla \times \overrightarrow{\mathbf{G}}_{A} \cdot \mathbf{J}$ can be expanded in the following form

$$
\nabla \times \overrightarrow{\mathbf{G}}_{A} \cdot \mathbf{J}=\nabla G_{A}^{x x} \times J_{x} \hat{x}+\nabla G_{A}^{y y} \times J_{y} \hat{y}+\nabla G_{A}^{z z} \times J_{z} \hat{z}
$$


Therefore, in the solution of MPIE via MoM, one needs to calculate the gradient of the potential GF which may be obtained simply by taking the gradient of the Ewald representation (7), yielding $\nabla \Psi=\nabla \Psi_{1}+\nabla \Psi_{2}$, where

$$
\begin{gathered}
\nabla \Psi_{1}=\frac{\mathrm{j}}{V} \sum_{m, n, p} \frac{\mathrm{e}^{-\frac{\gamma_{m n p}^{2}}{4 E^{2}}}}{\gamma_{m n p}^{2}} \mathbf{K}_{m n p} \mathrm{e}^{\mathrm{j} \mathbf{K}_{m n p} \cdot \mathbf{R}} \\
\nabla \Psi_{2}=\frac{1}{8 \pi} \sum_{m, n, p} \mathrm{e}^{-\mathrm{j} \mathbf{k} \cdot \mathbf{r}_{m n p}}\left(\frac{4 E}{R_{m n p} \sqrt{\pi}} \mathrm{e}^{-R_{m p p}^{2} E+\frac{k^{2}}{4 E^{2}}}+\sum_{ \pm} \frac{-1 \pm \mathrm{j} k R_{m n p}}{R_{m n p}^{2}} \mathrm{e}^{ \pm \mathrm{j} k R_{m n p}} \operatorname{erfc}\left(R_{m n p} E \pm \frac{\mathrm{j} k}{2 E}\right)\right) \frac{\mathbf{R}_{m n p}}{R_{m n p}}
\end{gathered}
$$

with both series $\nabla \Psi_{1}$ and $\nabla \Psi_{2}$ keeping the Gaussian convergence rate.

\section{EXTRACTION OF THE SINGULAR TERM}

Potential GFs have singularity of $1 / R$ type. Since only one term $(m=n=p=0)$ of the image sum $\Psi_{2}$ (12) is singular, if one extracts the singularity from this term, then the remaining series are all regular with finite value everywhere. To extract this singularity, one can add and subtract a $1 /\left(4 \pi R_{000}\right)=1 /(4 \pi R)$ term so that $\Psi=\Psi_{1}+\left(\Psi_{2}-1 /(4 \pi R)\right)+1 /(4 \pi R)$. Note that $1 / R$ can be integrated analytically over the linear basis functions [18], [19] and the remaining part has a finite value at $R \rightarrow 0$ since:

$$
\lim _{R \rightarrow 0}\left(\sum_{ \pm}\left(\frac{\mathrm{e}^{ \pm \mathrm{j} k R}}{R} \operatorname{erfc}\left(R E \pm \frac{\mathrm{j} k}{2 E}\right)\right)-\frac{2}{R}\right)=\mathrm{j} k\left(\operatorname{erfc}\left(\frac{\mathrm{j} k}{2 E}\right)-1\right)-\frac{2 E}{\sqrt{\pi}} \mathrm{e}^{\frac{k^{2}}{4 E^{2}}} .
$$

In (20), on the other hand, the only singular term corresponds to $m=n=p=0$ and it is in the form of $R^{-2}$. Adding $\mathbf{R} /\left(4 \pi R^{3}\right)$ to this term, after some algebraic efforts, one obtains:

$$
\lim _{R \rightarrow 0}\left(\frac{4 E}{R \sqrt{\pi}} \mathrm{e}^{-R^{2} E^{2}+\frac{k^{2}}{4 E^{2}}}+\sum_{ \pm}\left(\frac{-1 \pm \mathrm{j} k R}{R^{2}} \mathrm{e}^{ \pm \mathrm{j} k R} \operatorname{erfc}\left(R E \pm \frac{\mathrm{j} k}{2 E}\right)\right)+\frac{2}{R^{2}}\right)=-2 k^{2} .
$$

This leads to a smooth function that has finite values everywhere and can be integrated numerically, while the integration of the above singular term over basis functions can be carried out analytically [18]-[20].

\section{NUMERICAL EXAMPLES}

Consider a skewed lattice defined with vectors: $\mathbf{a}_{1}=a \hat{x}, \quad \mathbf{a}_{2}=0.5 a(\hat{x}+\sqrt{3} \hat{y})$, and $\mathbf{a}_{3}=0.5 a(\hat{y}+\sqrt{3} \hat{z})$. Let the source position be fixed at $\mathbf{r}^{\prime}=\left(\mathbf{a}_{1}+\mathbf{a}_{2}+\mathbf{a}_{3}\right) / 2=\mathbf{d} / 2$ and the observer be at the distance $R$ from the source along the diagonal $\mathbf{r}=\tau \mathbf{d}, \tau \in[0,1]$ (Fig. 1). The number of significant digits of accuracy in the computed Ewald sums as the number of terms grows is presented in Fig. 2. The reference value for estimating the accuracy was computed for a large number of terms in both sums (3375). We can observe a very rapid convergence rate for small and $\lambda$-periodic spacing in both cases, when the source is close to the observer ( $R=0.01 d$ ), and close to the edge of the unit cell ( $R=0.4 d$ ). Already for 125 terms $(m, n, p=-2, \ldots 2)$, both sums achieve more than 8 digits of accuracy as compared to the reference value. Fig. 3(a) illustrates the case of a 
large periodic spacing and the significant loss of accuracy that occurs due to a finite machine precision when a balanced optimal value of the parameter $E$ is used. However, when the parameter $E$ is based on the maximum permitted exponent, the accurate values of the GF are obtained [Fig. 3(b)]. In that case the sums are unbalanced and the modal sum needs much more terms to converge (Fig. 4).

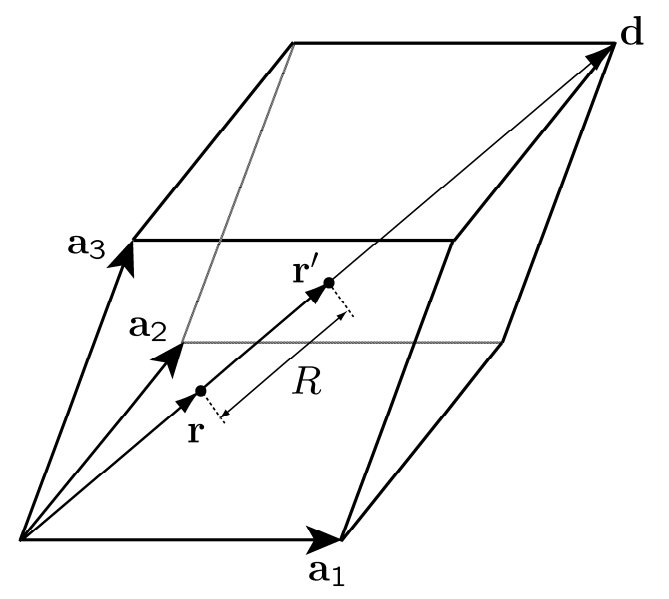

Fig. 1. The unit cell of a skewed 3-D lattice.
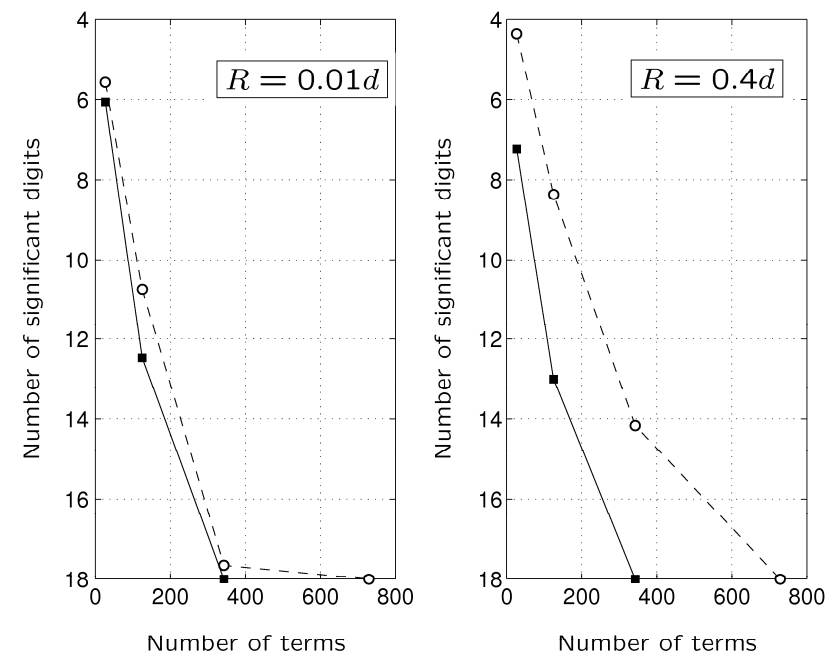

(a)
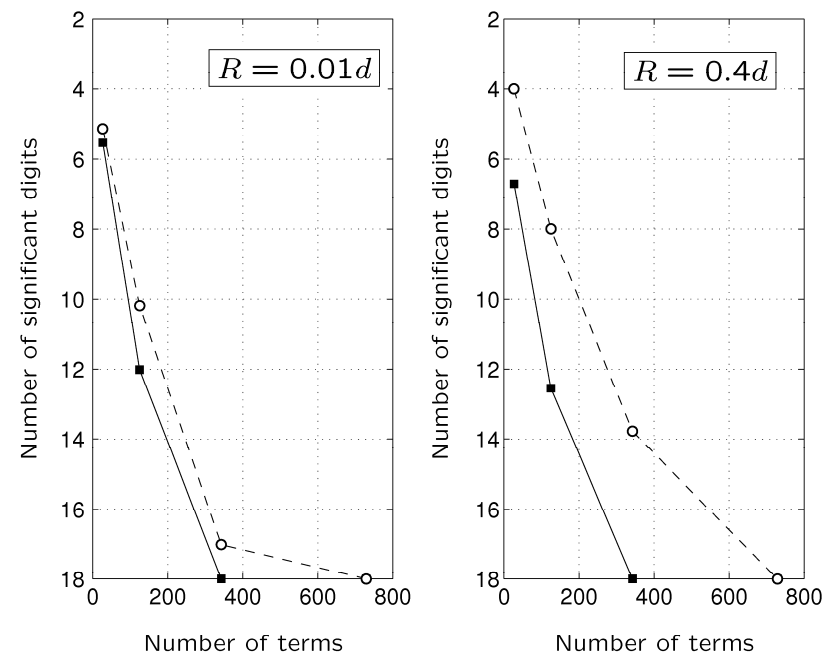

(b)

Fig. 2. The accuracy expressed in the number of significant digits as compared to the reference value vs. the number of terms. The splitting parameter corresponds to the optimal value $E / E_{0}=1$ (16). The reference value taken for $N=(2 \cdot 7+1)^{3}=3375$ terms. Solid lines $(\square)$ represent the convergence of the modal sum $\Psi_{1}$ and the dashed ones (O) of the image sum $\Psi_{2}$. (a) $a=\lambda / 5$ and (b) $a=0.99 \lambda$. 


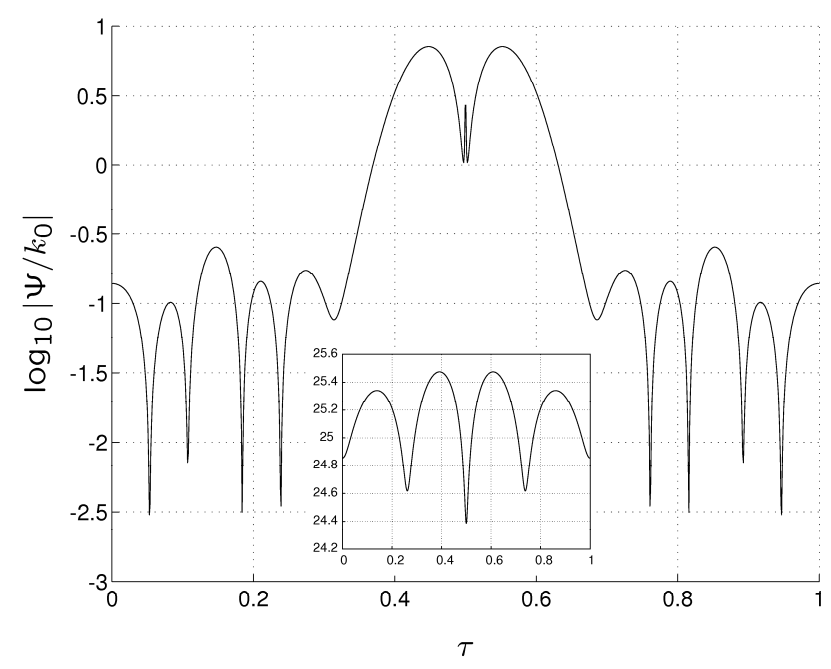

Fig. 3: The normalized GF $\Psi$ along the diagonal $\mathbf{d}$ for large periodic spacing $a=5 \lambda$. If the splitting parameter $E / E_{0}=2.78$ is based on the maximum permitted exponent $\left(H^{2}=9\right)$, accurate values of the GF are obtained. Inset shows a significant loss of accuracy due to a finite machine precision, when the splitting parameter corresponds to the optimal balanced value $E / E_{0}=1$.

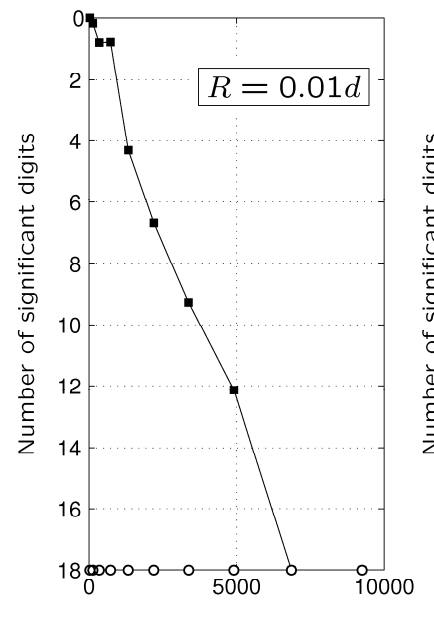

Number of terms

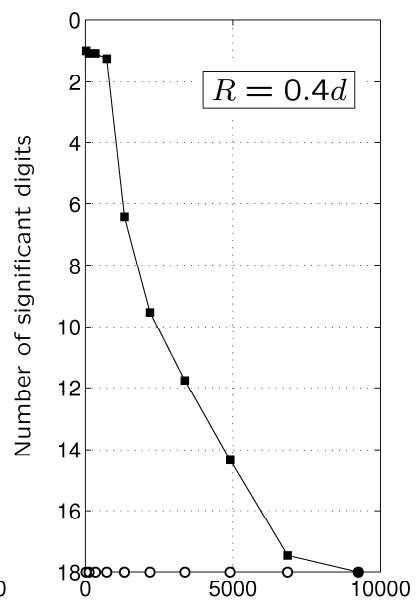

Number of terms

Fig. 4. Large periodic spacing $a=5 \lambda$. The accuracy expressed in the number of significant digits as compared to the reference value vs. the number of terms. The splitting parameter $E / E_{0}=2.78$, satisfies $H^{2}=9$. The reference values taken for $N=(2 \cdot 13+1)^{3}=19683$ terms. The lines with squares (回) represent the convergence of the modal sum $\Psi_{1}$ and the lines with circles $(\mathrm{O})$ of the image sum $\Psi_{2}$.

\section{TRIPLY PERIODIC ARRAY OF METALLIC CUBES}

In the determination of the dispersion diagram there is no incident field and the analysis leads to a homogeneous problem. When using the IE method, the matrix of the resulting homogeneous problem depends on both the propagation constant and the frequency, and it is not possible to separate the two dependencies. In this case, for a given value of the propagation constant, frequency iteration is needed to find the nontrivial solutions of the problem and the resulting points of the dispersion diagram. In this work we have used a strategy based on the search of the minimum singular value of the MoM matrix determinant [21]. 
We consider a 3-D array of perfectly conducting metallic cubes whose unit cell is depicted in inset of Fig. 4. The mesh consisted of 506 triangular basis functions. The wave vector $\mathbf{k}$ was changed in the following manner: for $\mathrm{A} \rightarrow \mathrm{B}: 0 \leq k_{x} \leq \pi / a_{1}, k_{y}=k_{z}=0 ; \mathrm{B} \rightarrow \Gamma: k_{x}=\pi / a_{1}, 0 \leq k_{y} \leq \pi / a_{2}$, $k_{z}=0$; and for $\Gamma \rightarrow \Delta: k_{x}=\pi / a_{1}, k_{y}=\pi / a_{2}, \quad 0 \leq k_{z} \leq \pi / a_{3}$. The incremental step between successive calculation points was $\pi / 10$. The accuracy of the results obtained using an IE-MoM technique with the GF presented in this work has been confirmed by comparison with the finiteelement results reported in [22].

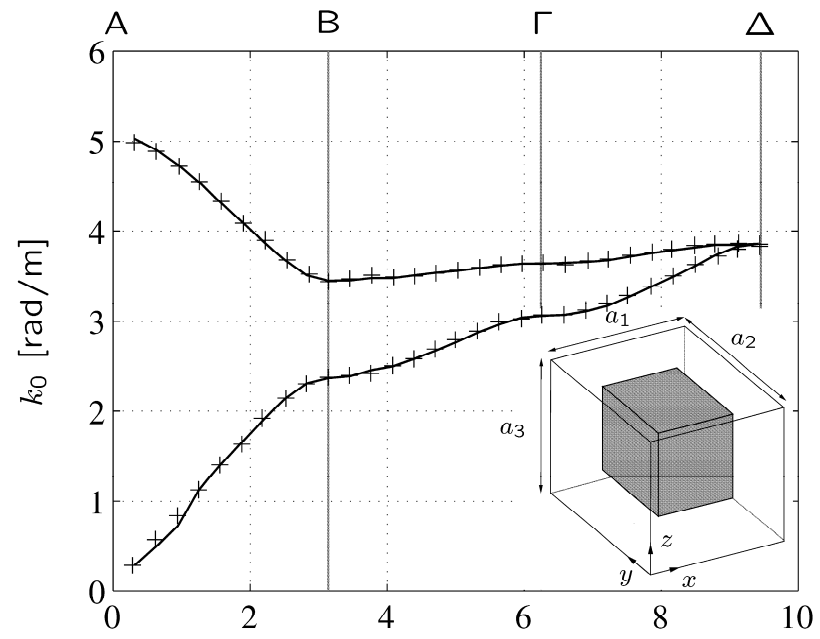

Fig. 5. Dispersion diagram of triply periodic array of perfectly conducting metallic cubes. Our simulation (solid lines) compared to the FEM simulation taken from [22] (+). The inset shows a unit cell of a triply periodic array of metallic cubes. Orthogonal lattice has the following parameters $a_{1}=a_{2}=a_{3}=1 \mathrm{~m}$. The metallic cube has all sides $w=0.5 \mathrm{~m}$ and is centered in the unit cell. The structure is in the air.

\section{CONCLUSION}

The current increase of possible applications for periodical structures like electromagnetic/photonic band-gap materials and generic metamaterials based on periodic lattices makes desirable to enlarge the scope of the corresponding numerical analysis tools. In the framework of the integral equation model for periodic structures, orthogonal lattices have been successfully tackled with the Ewald transformation. This paper has generalized it to periodic 3-D skewed lattices. This enlarges the scope of applicability of the Ewald transformation and adds extra flexibility to represent real-life and useful geometries. The choice of the splitting parameter has been discussed and the optimum value for 3-D skewed lattices with small and large periodic spacing has been derived. The resulting sums have Gaussian convergence and only a few terms are needed to achieve a good accuracy. The convergence properties have been analyzed analytically and numerically and the singularity extraction has been addressed. All these are necessary steps when solving periodic printed structures (infinite arrays) with a mixed potential integral equation. Finally, we have validated the developed technique by comparing its numerical predictions with results from an FEM based solver.

\section{REFERENCES}

1. Special issue on electromagnetic crystal structures, design, synthesis, and applications, IEEE Trans Microwave Theory Tech 47 (1999).

2. Special issue on metamaterials, IEEE Trans Antennas Propag 51 (2003). 
3. N. Kinayman and M. I. Aksun, Comparative study of acceleration techniques for integrals and series in electromagnetic problems, Radio Sci 30 (1995), 1713-1722.

4. P. P. Ewald, “Die Berechnung Optischer und Elektrostatischer Gitterpotentiale,” Ann Phys 64 (1921), $253-287$.

5. C. M. Linton, The Green's function of the two-dimensional Helmholtz equation in periodic domains, J Eng Math 33 (1998), 377-402.

6. F. Capolino, D. R. Wilton, and W. A. Johnson, Efficient computation of the 2-D Green's function for 1-D periodic structures using the Ewald method, IEEE Trans Antennas Propag 53 (2005), 2977-2984.

7. A. W. Mathis and A. F. Peterson, Efficient electromagnetic analysis of a doubly infinite array of rectangular apertures, IEEE Trans Microwave Theory Tech 46 (1998), 46-54.

8. K. E. Jordan, G. R. Richter, and P. Sheng, An efficient numerical evaluation of the Green's function for the Helmholtz operator on periodic structures, J Comput Phys 63 (1986), 222-235.

9. T. M. Grzegorczyk, C. D. Moss, J. Lu, X. Chen, J. Pacheco, Jr, and J. A. Kong, Properties of left-handed metamaterials: transmission, backward phase, negative refraction, and focusing, IEEE Trans Microwave Theory Tech 53 (2005), 2956-2967.

10. I. Stevanović, P. Crespo-Valero, K. Blagović, F. Bongard, J. R. Mosig, Integral equation analysis of 3-D metallic objects arranged in 2-D lattices using the Ewald transformation, IEEE Trans Microwave Theory Tech 54 (2006), 3688-3697.

11. M.-J. Park, J. Park, and S. Nam, Efficient calculation of the Green's function for the rectangular cavity, IEEE Microwave Guided Wave Lett 8 (1998), 124-126.

12. A. Borji and S. Safavi-Naeini, Fast convergent Green's function in a rectangular enclosure, Proc IEEE Antennas Propag Int Symp, Columbus, OH, 2003, pp. 950-953.

13. F. S. Ham and B. Segall, Energy bands in periodic lattices-Green’s function method, Phys Rev 124 (1961), 17861796.

14. A. Kustepeli and A. Q. Martin, On the splitting parameter in the Ewald method, IEEE Microwave Guided Wave Lett 10 (2000), 168-170.

15. M. Abramowitz and I. A. Stegun, Handbook of Mathematical Functions with Formulas, Graphs, and Mathematical Tables, Dover, New York, 1972, pp. 295-329.

16. G. P. M. Poppe and C. M. J.Wijers, More efficient computation of the complex error function, ACM Trans Math Softw 16 (1990), pp. 38-46.

17. IMSL Fortran Subroutines for Mathematical Applications. Visual Numerics Inc., Houston, TX, (1997), http://vni.com

18. D. R. Wilton, S. M. Rao, A. W. Glisson, D. H. Schaubert, O. M. Al-Bundak, and C. M. Butler, Potential integrals for uniform and linear source distributions on polygonal and polyhedral domains, IEEE Trans. Antennas Propag, AP-32 (1984), 276-281.

19. R. D. Graglia, On the numerical integration of the linear shape functions times the 3-D Green's function or its gradient on a plane triangle, IEEE Trans Antennas Propag 41 (1993), 1448-1455.

20. P. Ylä-Oijala and M. Taskinen, Calculation of CFIE impedance matrix elements with RWG and $n \times R W G$ functions, IEEE Trans Antennas Propag 51 (2003), 1837-1846.

21. V. A. Labay and J. Bornemann, Matrix singular value decomposition for pole-free solutions of homogeneous matrix equations as applied to numerical modeling methods, IEEE Microwave Guided Wave Lett 2 (1992), 49-51. 
22. C. Mias, J. P. Webb, and R. L. Ferrari, Finite element modelling of electromagnetic waves in doubly and triply periodic structures, IEEE Proc Optoelectron 146 (1999), 111-118. 